\title{
The effectiveness of providing autologous transfusion education to operating room staff
}

\author{
Azita Chegini ${ }^{1}$, Maryam Zadsar ${ }^{1 *}$, Mojgan Shaiegan ${ }^{1}$ \\ Received: 28 Mar 2017 \\ Published: 18 Dec 2017 \\ Copyright $@$ Iran University of Medical Sciences
}

Cite this article as: Chegini A, Zadsar M, Shaiegan M. The effectiveness of providing autologous transfusion education to operating room staff. Med J Islam Repub Iran. 2017 (18 Dec);31:101. https://doi.org/10.14196/mjiri.31.101

\section{Brief communication}

Transfusion of blood components can save lives; however, it may pose some risks, or cause morbidity or mortality. Theoretical and practical knowledge is needed to ensure patients' safety in transfusion. The level of transfusion medicine education varies in different countries (1). Nowadays, there are defects in transfusion medicine education and its curricula, thus, it needs to be reviewed and improved (2,3). Despite improvements in the medical science and technology, in developing countries, transfusion medicine education is still insufficient and based on lecture-oriented method (4). Lack of awareness and knowledge in this field could have dangerous consequences for patients and decrease their safety (2). Typically, physicians are responsible for transfusion, but nurses are assigned for the care of patients, which is an essential part of clinical practice, such as injection and transfusion safety. Thus, it is highly important to provide more advanced education courses on basic issues of transfusion sciences for nurses and physicians.

However, there are published health services curricula for better transfusion approaches (4). The National Patient Safety Agency (NPSA) considered the requirements of personnel training and right blood to the right patient program (5-7). Several studies have been conducted about prescription, documentation, bedside administration, and the clinical practice of transfusion, which could improve physicians' skills through training and formal education (6). In order to decrease transfusion- transmitted infections, especially new pathogens, minimizing utilization of allogeneic blood has become a desirable goal. Knowledge and awareness of the operating room staff about autologous transfusion in one hospital in Tehran was evaluated to improve this plan, and it was tried to promote the knowledge of practitioners through a short-term education.

A total of 27 operating room staff participated in a short educational course on autologous transfusion and its related indications, contraindications, the method of procedures, advantages, disadvantage, and adverse effects. The participants with the least working experience of 15 years were selected from the staff of the operating room from one hospital. However, 3 of them did not complete the process and were excluded from the study. The model of team- based learning and feedback of the correct answers was applied. A standard questionnaire was designed and validated based on the lessons' content and training objectives $(8,9)$.

The participants' score of knowledge was assessed by the standard questionnaire before and after the end of the course. A total of 12 questions were about the knowledge of trainees about autologous transfusion. The questions were categorized into 4 classes: definition, methods, indications, contraindications, and advantages. Data of the correct peer pre- and posttest answers for each precipitant from each category were collected and analyzed using SPSS Version 18 (Chicago IL). The mean and standard deviation of the pre- and posttest final scores were compared by paired t test. The frequency of the correct answers of pre- and post-education exams was also reported based on the category of the questions. The demographic data and academic degree of the participants were evaluated with respect to their exam score.

In total, 24 operating room staffs were enrolled in the study. The mean of the pretest correct answers was $32.64 \% \pm 18$, and the mean of posttest correct answers was $75 \% \pm 17(\mathrm{p}<0.001)$. The minimum and maximum of the pretest correct answers varied from $0 \%$ to $75 \%$, and the same measures for posttests varied from $42 \%$ to $100 \%$. The mean of improvement in knowledge of the trainees was $5.08 \pm 3.36$.

The mean age of the participants was $44 \pm 8.2$; noticeably, the mean of working experience was $20 \pm 5.7$ years; 9 persons were anesthetic nurses, 7 physicians, and 8 were 
operation room nurses.

The worst correct answers score was found in the Question 8 by just 2 correct answers in the pre-education exam, which raised up to 14 correct answers in the post education exam, showing an improvement score of 9, which was near 2 times more than the mean of improvement score of the present study (Graph 1).

The mean of improvement score in the post-education exam was $10 \pm 3.18$. Improvement in the scores for Questions $5,7,12$, and 9 found to be below average.

The mean of improvement score in the indication of autologous transfusion was $10 \pm 3.6$; the given score for the questions about contraindication of autologous transfusion was $6 \pm 1.4$, and the same score for the questions about the methods of autologous transfusion was 11.5 \pm 4.04 . Knowledge of the participants about the advantages and definition of autologous transfusion gained the mean improvement score of $10.6 \pm 1.15$.

The data on the numbers and percentages of correct answers pre- and post- education are presented in Table 1.

To promote the patient blood management program in Iran, we designed a package for educating about autologous transfusion containing definition, indications, contraindications, advantages and risks presented through lectures, power point, images and films by team-based learning method and feedback from the tests. The minimum and maximum of the pretest correct answers varied from $0 \%$ to $75 \%$. The same measures for posttests were found from $42 \%$ to $100 \%$. The mean of improvement of knowledge in trainees was 5.08 \pm 3.36 .

As it is described by Accreditation Council for Graduate Medical Education (ACGME), transfusion medicine is a scientific subject, which has started from donor selection to the end of transfusion chain, utilization, and blood alternatives (10). In every blood service, level of physician knowledge plays a principle role in modern health care. Previous studies have found that medical directors play an important role in solving the problems related to blood products and education needs. In 2007, the Academy of Clinical Laboratory and Physicians and Scientists (ACLSPs) introduced an education program for residents, which included lectures, reading, discussions, conferences, journal clubs, individual training, and exams (11). The next strong step was taken in 2009 , by implementing a fellowship course for the pathologists, hematologists, internal medicine specialists, pediatricians, obstetrics \& gynecologists, and anesthesiologists (12). O'Brien described a deep gap of transfusion medicine knowledge in medical school graduates who wanted to begin residency (3). Radical changes had occurred in the field of transfusion medicine during the last 50 years. Thus, updating education systems and materials is an essential requirement. An original research, which tried to develop and evaluate a tool for assessing the skill and knowledge competency of staff involved in blood transfusion, found that safe transfusion practice is a complex, multi-step process, and consequently, there is potential for error occurring at each stage. Therefore, the ability of staff to maintain associated knowledge and skills during the working years is highly important. Finally, they concluded that a collaborative approach is necessary to develop an effective professional career (13).

Transfusion medicine education in Iran is highly subtle, and there is a large need to train post- graduate practitioners. Thus, we planned an educational autologous transfusion program, which plays an essential role in promoting the patient blood management program. As it was found by Jan Graham et al., competency and awareness of junior doctors was associated with their undergraduate education in transfusion and attendance in clinical wards. A total review of courses was assessed positively by participants (6).

For ongoing education, several methods have been suggested, such as one by one meeting for physicians, prescheduled conferences, and daily clinical rounds on transfused patients, reviewing the orders of transfusion, providing and encouraging the implemention of the guidelines and algorithms.

The model of team- based learning and feedback of correct answers was applied. A standard questionnaire was designed and validated based on lesson content and training objectives. The participants ' score of knowledge was assessed by the standard questionnaire before and after the end of the course. A total of 12 questions on knowledge of trainees about autologous transfusion were applied. The mean of the pretest correct answer was $32.64 \% \pm 18$, and the mean of posttest correct answers was $75 \% \pm 17(\mathrm{p}<0.001)$.

Finally, we found that the basic knowledge of operating room staff was very slight although a cross-sectional short-term team- based learning can improve transfusion knowledge.

Transfusion medicine training needs to be critically revised for the clinical staff. Moreover, ongoing short- term training courses can promote the knowledge of transfusion of the involved personnel about patient blood management, and thus, improve patients' safety.

\section{References}

1. Panzer S, Engelbrecht S, Cole-Sinclair M, Wood E, Wendel S, Biagini $\mathrm{S}$, et al. Education in transfusion medicine for medical students and doctors. Vox Sang. 2013;104(3):250-72.

2. Strauss RG. Transfusion medicine education in medical school: only the first of successive steps to improving patient care Transfusion. 2010;50(8):1632-5.

3. O'Brien KL, Champeaux AL, Sundell ZE, Short MW, Roth BJ. Transfusion medicine knowledge in postgraduate year 1 residents. Transfusion. 2010;50(8):1649-53

4. Shander A, Hofmann A, Ozawa S, Theusinger OM, Gombotz H, Spahn DR. Activity-based costs of blood transfusions in surgical patients at four hospitals. Transfusion. 2010;50(4):753-65.

5. Mole LJ, Hogg G, Benvie S. Evaluation of a teaching pack designed for nursing students to acquire the essential knowledge for competent practice in blood transfusion administration. Nurse Educ Pract. 2007;7(4):228-37.

6. Graham J, Grant-Casey J, Alston R, Baker P, Pendry K. Assessing transfusion competency in junior doctors: a retrospective cohort study. Transfusion. 2014;54(1):128-36.

7. Gharehbaghian A, Javadzadeh Shahshahani H, Attar M, Rahbari Bonab M, Mehran M, Tabrizi Namini M. Assessment of physicians knowledge in transfusion medicine, Iran, 2007. Transfusion Med. 2009 Jun 1;19(3):132-8.

8.Hijji BM, Oweis AE, Dabbour RS. Measuring knowledge of blood transfusion: A survey of Jordanian nurses. Am Int J Contemp Res. 
2012;2:77-94

9.Aslani Y, Etemadyfar S, Noryan K. Nurses' knowledge of blood transfusion in medical training centers of Shahrekord University of Medical Science in 2004. Iran J Nurs Midwifery Res. 2010;15(3):141.”

10. Brotherton SE, Etzel SI. Graduate medical education, 2008-2009. JAMA. 2009 Sep 23;302(12):1357-72.

11. Wu Y, Tormey C, Stack G. Resident and fellow training in transfusion medicine. Clin Lab Med. 2007;27(2):293-342.

12. Alexander CB. Pathology graduate medical education (overview from 2006-2010. Hum Pathol. 2011;42(6):763-9.

13. Pirie E, Gray M. Exploring the assessors' and nurses' experience of formal assessment of clinical competency in the administration of blood components. Nurse Educ Pract. 2007;7(4):215-27. 